\title{
Maintaining genetic variation in breeding popu- lations of Radiata pine in New Zealand
}

\author{
C.J.A. Shelbourne
}

NZ Forest Research Institute Ltd., Rotorua, New Zealand, 99 Acacia Road, RD 5, Rotorua 3076, New Zealand E-mail: cjashelbourne@hotmail.com

\begin{abstract}
Advanced generation selection (AS) for the future breeding population (BP), becam a focus of tree breeders' thinking in the mid 1970s., particularly with Pinus radiata in New Zealand (NZ). Multitrait selection among families was generally recommended, but this reduced genetic variation in the future breeding population.

From Shaw and Hood's (1985) stochastic simulation, later confirmed by Rosvall, Lindgren and Mullin's (1998) stochastic simulation on Norway spruce, it was realised that selecting within families rather than among families of a new breeding population avoided any reduction of genetic variation in the BP. Heritabilities were low for seedling within-family selection but clonal replication within families should strongly increase heritabilities. Gains from cloned versus seedling populations of equal numbers of plants were also deterministically simulated (Shelbourne et al. 2007), and balanced (within-family) selection gains from the cloned populations were all higher than seedling equivalents at heritabilities of 0.5 and under.

The late P.A. Jefferson's (2016) Breeding Management Plan (which will be soon superceded) contains a re description of New Zealand (NZ) radiata pine breeding. Selections were made in crosses from the earlier program and OP see and scion material were collected from all 360 selections. OP family tests of selections have been planted at 11 sites in NZ and 7 in New South Wales and Tasmania, and scions of their female parents have all been grafted at an archive. Crosses made in the archive are being cloned and the programme was committed to within-family selection to retain genetic variance for the future closed breeding population.

Clonally-replicated testing paired with within-family selection is the solution for balancing long-term gain and diversity in BP and PP.
\end{abstract}

Keywords: : Pinus radiata, genetic variation, breeding population, within-, among-family selection

\section{Introduction}

This review is about maintaining genetic variation in a breeding population, particularly the New Zealand radiata pine breeding population, in the face of the loss of this variation through among-family selection and backwards selection. The history of the radiata pine breeding from its initiation in 1952 to the present is given in a book by Shelbourne and Carson which is being published by Springer Nature. The story starts with Libby (1964) on clonal selection and ends with the late Paul Jefferson's Breeding Management Plan in 2016.

\section{Breeding strategy development and cloning}

The Libby paper is probably the first that covers clonal testing in a forestry context, but examines this only for genetic testing of seed orchard clones. It incorporates quantitative genetic theory from animal breeding, which uses a combined index of family and individual forwards selection for predicting genetic gain.

Libby showed relative efficiency values for a variety of heritabilities and family sizes and in all cases clonal selection was more efficient than family-plus-mass selection which in turn was more efficient than family or mass selection. If genotype $\mathrm{x}$ environment interaction was strong, clonal testing at several sites was necessary. However Libby did not address the question of loss of genetic variation in among- versus withinfamily selection in breeding population.

Namkoong, Snyder and Stonecypher (1966) presented the first clear statement of gain prediction equations for different selection and seed orchard scenarios for tree breeding, based on the pine programs of the south-east USA. They focused on gains from seed orchards (the production population, PP), particularly on the clonal versus seedling orchard controversy of the era. Orchards were regarded as an integral part of the breeding cycle and strategy. Namkoong et al's' generalised equations are adaptable to most selection situations of breeding populations and seed orchards. They did not give models for 
clonal forestry or clonal testing, though clonal testing is mentioned as an alternative to progeny testing, without gain expectations.

Shelbourne (1969) used Namkoong et al.'s predicted gain for RS/GCA to add gain for a 'Clonal test for GCA'. Gain was predicted for phenotypic clonal selection plus selection on clone means using a clonal test. The latter is the gain from clonewithin-family selection, and the basis for subsequent examples (and applies to any genetically-variable and vegetativelypropagatable species).

In the first clonal test of PR in New Zealand (Shelbourne and Thulin 1974), half the ortets were selected for high wood density in un-improved 5- and 6-year-old stands. Growth of cuttings was good, though less than seedlings, but their morphology showed effects of maturation. At 4 years from planting, broad sense heritabilities $\left(\mathrm{H}^{2}\right)$ for various traits were around 0.40 and heritabilities of clone means, $0.71-0.86$, based on 9 ramets/clone. Their relatively high level of maturation and inadequate re-propagation was a barrier to clonal forestry and for cloning a breeding population.

Burdon and Shelbourne (1971) were the first to define a 'breeding population' (BP). Various mating designs were compared for Advanced Generation Selection (AS), such as openpollinated (OP), North Carolina II (NC2), diallel (D) and singlepair-mating (SPM). OP family means were only used for backwards selection of parents. They recognised that the best information for selection of individuals within families would be from clonal replication of seedlings, with an advantage in gain at lower heritabilities. They proposed using OP families for initial GCA estimation, with cloned SPM families (backwards selected) for within-family selection. Cloning OPs was not considered as a main-stream strategy, partly because propagating sufficient juvenile ramets per cloned seedling of radiata for a clonal test was not yet feasible. They also recognised that the trade-off of seedling-within-family selection intensity against number of ramets per clone could result in poorer family mean estimation. Cloning was found to be invaluable in selection for stability across sites where there is marked genotype $x$ environment interaction (GxE). There was no recognition of the maintenance of genetic variance in the breeding population by within-family selection.

A robust and complete model for cloning (Burdon and Shelbourne 1974) included maternal effects of clones, effects of individual ramets, and environments, with all the interactions. They also compared the variance structures of half-sib, full-sib and cloned families, and highlighted the value of cloning for advancing BPs, including screening environments for GxE. There are two types of information modeled; genotypic values of individuals, and genetic parameters, including variances and covariances among traits. This model supports the replication of clones in within-family selection but there was no overt recognition of the loss of genetic variation in amongversus within-family selection .

At the 1977 Rotorua conference on Advanced Selection Strategies there was some confusion about the respective roles of General Combining Ability (GCA) ranking and advanced generation family (AS) ranking. A multi-trait combined index, used for radiata by Wilcox (Burdon, Shelbourne and Wilcox 1977) and by Baradat in France (covered by Stonecypher and Arbez in Bordeaux in 1976), involved too much amongfamily selection to avoid reduction of genetic variation. There was no mention of within-family versus among-family selection, and genetic variation.

Shaw and Hood's (1985) paper provided an early alert on the benefit of within-family versus among-family selection in maintaining genetic variation in the breeding population. They constructed a model for stochastic simulation and found that using clonal replication i avoided increased relatedness, and selecting within families in breeding populations versus selecting among them was still effective in generating gain. .

Rosvall, Lindgren and Mullin's (1998) paper on Norway spruce was about stochastic simulation of the use of cloning in within-family selection, and later in this review (1998), provided results of a 10-generation simulation of within-family clonal selection that was highly relevant to radiata pine breeding in NZ.

\section{Selecting among versus within families in the breeding population}

The Development Plan (Shelbourne, Burdon, S.D. Carson, Firth and Vincent 1986) addressed various aspects of breeding strategy relevant to within-family selection and cloning. The new control-pollinated seed orchard, only started at Amberley in 1984, was a strategy-changing innovation. The future success of CP orchards was correctly seen as dependent on the development of cutting propagation from nursery stools to multiply stock from expensive seeds. The main conflict in breeding populations was between using within- versus among-family selection for maximum gain but with increased relatedness. Only within-family selection, avoids reduction in additive genetic variance and increased inbreeding. So the best strategy is to use mainly within-family selection for the breeding population and between-family or between-parent selection for the orchards.

The 1986 plan for advanced generation selection (AS) of the BP was to plant single CP family blocks of 50 seedling trees to increase gain from within-family selection. Applying cloning in single-family blocks would be very advantageous for withinfamily selection, but the possibilities for using rooted cutting clones, planted on several sites, for forwards selection (within families) remain to be explored'. In 1986 the need for withinfamily selection in the BP was recognised but the technology for producing maturation-free cuttings in quantity was not yet available.

Among-family selection for GCA therefore would cause decreased genetic variance in the breeding population, but for the production population (seed orchards), among-family selection was needed to select the best parents and maximise gain. Cloned forwards within-family selection would contribute gain for the new orchard clones as well as the breeding population. Within-family cloning was dependent on producing sufficient maturation-free cuttings from one seedling in one year. 
It was clear from deterministic simulation (Shelbourne 1992) that low intensity among-family selection in the BP, was not going to give appreciable gains each generation, yet solely within-family selection combined with cloning could facilitate high heritability selection done within family.

A research clonal trial at two sites (Kumar 2006) verified that clone means were strongly related to the clone's offspring breeding value (BV). This trial, originating in 1984, was based on 20 full-sib families and 10 clones per family, and utilized fascicle cuttings raised in the greenhouse. The clones retained their juvenile state at time of planting, and because there were several clones per family, this confirmed the potential gain of clone-in-family forwards selection. Later their open-pollinated offspring showed that their BVs correlated well with those of the parent clones. The correlations between clonal means and their OP progeny means were 0.56 (dbh), 0.63 (straightness), 0.81 (branch cluster number), 0.09 (malformation) which all depended largely on the heritability of the trait in the OP test. Clonal performance of selections in Main and Elite BPs could thus be used for direct selection in seed orchards (and for AS) with no need to do progeny testing.

\section{Genetic variance and within-family selection}

Burdon and Namkoong (1983) in their paper on multiple populations and sublining raised two problems, without any immediate solution. These were uncertainty about future economic weights of prospective selection criteria and the question of future inbreeding and narrowing of the genetic base from selecting in closed populations. This was the first indication that a solution was needed for maintaining genetic variance in the BP.

Rowland Burdon in 1989 in his paper on early selection warned that the shorter the generation time, the quicker the breeding population may 'burn up' its genetic variation, as effective population size will be run down each generation, especially if family selection is involved.

In a deterministic simulation of gains of various BPs, Shelbourne (1992) concluded that genetic gains from different kinds of breeding populations and seed or plant production populations showed that even low intensity among-family selection in the $\mathrm{BP}$, was not going to give much gain in the BP each generation. Solely within-family selection combined with cloning could facilitate high heritability selection within family, and increase BP gain.

The stochastic simulation study by Gea et al. (1997) did not address the genetic variance situation in the BP directly but provided some information about the choice of size of subgroups of breeding populations. Although the use of small disconnected groups over many generations was better at preserving status number than large groups, inbreeding in the small groups got too severe in the smallest ones, and gains were bigger in the large groups. Small groups would not be a good long-term strategy. Small groups were fine for a strategy of very few generations.

Cloned multiple populations were being used in the Swedish breeding population of Norway spruce (Danell 1993), and clonal testing proved a good alternative to progeny testing as a means of identifying good genotypes. Clonal testing appeared much more accurate; $1 / 4$ the number of test trees were needed, but that there were some risks of maturation, causing " $c$ " effects. Long term gains were highest for balanced within-family clonal selection.

The Rosvall, Lindgren and Mullin (1998) paper on Norway spruce was about stochastic simulation of the use of cloning in within-family selection and this was highly relevant for radiata pine breeding. Stochastic simulation of balanced (ie. no among-family) selection enabled gain in the breeding and production populations to be tracked over 10 generations. There was no reduction in additive genetic variance in the BP for 10 generations from balanced within-family selection and clonal testing. This stochastic simulation involved a breeding population with 48 parents, 48 FS families, 40 clones/family and 14 ramets/clone. After 10 generations the linear increase in additive genetic effects was 11.0 sigma $A$ (square root additive genetic variance) and gain in a 6-clone seed orchard was 11.7 sigma $A$ (assuming $h^{2}$ of 0.2 ). Status number decreased from 48 to 8.1 in 10 years in the BP. Within-family selection on clone means with populations of 24 or more gave very low accumulation of inbreeding over 10 generations.

Jayawickrama and Carson 2000 documented the proposed new strategy for breeding PR. The strategy is based on additive genetic effects (RSGCA), and a 2-superline structure for Main population and Breeds (Elites). A non-regionalised BP is maintained, with final selection at about age 8 years. New breeds (Elites) are: Structural timber, Clear Cuttings, Growth and form, and Dothi-resistant. Combined population census number for breeds is $4 \times 24=96$. Census number of total population is 550 . The role of breeds is to get optimum gain while delaying inbreeding. The Main population is a reservoir of genetic diversity.

$\mathrm{OP}$, polycross and later NCII female tester were recommended entirely for GCA estimation. GCA ranking and recombination (AS) were therefore separated, though no explanation was given for the weighting given to among-family selection.

Cloning 10-20 seedlings from each breed family is planned. These are from control crosses for AS, to generate the next generation of the breed. Within-family selection should be more effective with clones-in-families.

The maintenance of genetic variance in the future BP was not well addressed in this breeding plan.

Another stochastic simulation (Burdon and Kumar 2004) of seed orchard gains from a breeding population showed gains from selection, ranked (lowest to highest) as Phenotypic, OP Forwards, OP Backwards, Polymix, Paircross. For $h^{2}=0.2$, gain was $1.98 \%$ for OP Forwards vs $2.32 \%$ for OP Backwards and $2.72 \%$ for Pair crosses. Gain from use of OPs for forwards selection may be compensated by much improved gain per unit time. Cloned within-family selection in OP, Polycross and Pair cross families would eliminate reduction in additive genetic variance in the $\mathrm{BP}$ and increase gains in $\mathrm{BP}$ and $\mathrm{PP}$.

Genetic gain was also deterministically predicted from one generation of balanced (no among-family) selection in a large Main OP breeding population for a range of 7 
heritabilities (Shelbourne et al. 2007). Gains from cloned versus seedling populations of equal numbers of plants were simulated, derived from forwards selection in open-pollinated, polycrossed and full-sisadb (pair-crossed) families. At $\mathrm{h}^{2} 0.5$ and below, the gain from cloned families was always higher than seedling families. Cloned polycross gains were highest, followed by cloned OP and then cloned full sib family gains. For example, at $\mathrm{h}^{2}$ of 0.3 , gain for OP seedlings was $9.1 \%$ versus OP clones of $12.1 \%$. Gains for all cloned BPs always exceeded those of seedlings up to and including heritability 0.5. OP seedlings and clones have a shorter breeding cycle than the others which gives them higher gains per year; gain per year for the cloned OP was nearly the same as for the cloned polycross.

Following these results, deterministically-simulated gains were compared of within-family selection of seedlings and clones (from the same number of plants) at $h^{2}$ of 0.2 and 0.5 (Shelbourne et al. 2006). Predicted gains for seedlings at heritabilities of 0.2 and 0.5 were 10.8 and $13.3 \%$. Predicted gains for clones were 13.2 and $17.9 \%$. For within-family selection, clones showed highly superior gains to seedlings up to and including a heritability of 0.5 .

\section{The breeding strategy for radiata pine in NZ and New South Wales}

Dungey et al. (2009) initially outlined the preceding strategy of Jayawickrama and Carson (2000), and then reported summaries of two lines of research by Shelbourne et al. (2007) and by Kumar (2006), commissioned by the RPBC.

The 'new' strategy (Dungey et al. 2009) consisted of a large open-pollinated seedling Main (MP) population with 500 female parents. The uncloned MP was to be managed in discrete generations, with forwards selection of the next generation in OP families, as well as some backwards selection in the present generation using previously GCA-tested parents. Inbreeding in the MP will be controlled by parental reconstruction and/or estimating group co-ancestry. Any inbreeding in the PP is contained within each subline and minimized by outcrossing in the CP orchards.

There would be a single small control-pollinated Elite population, (instead of 4, in Jayawickrama and Carson (2000)), planted half as seedlings and half as clones. The seeds of 48 parents will be propagated as clones and seed of a further 48 parents as seedlings, and they will be tested two-yearly following a Rolling Front establishment plan. Cloning gives relatively high heritability of clones-in-families for traits of lower heritability, while the seedling families are used for high heritability traits.

The Breeding Management Plan (Jefferson 2016) is not a publication and is summarised here from a draft copy I received soon after the sudden death of Paul Jefferson, the author. Following a meeting in October 2017, this has been superceded. The account of the existing genetic material is unaffected. This contains the latest description of NZ radiata pine breeding, and is different from the strategy proposed by Dungey et al. (2009). It describes the development of radiata pine breeding from 2003 until 2016.
NZ has long relied on progeny-tested clones in seed orchards for deployment of genetically-improved seed of radiata pine. In spite of good gains from the development of control-pollinated orchards, there had been little progress in breeding population (BP) development. Earlier controlled pollination, selection of plus trees, collection of their OP seed, establishment of progeny trials and clonal seed orchards had gone some way to progress the breeding population. The status number had been substantially reduced by strong amongfamily selection.

Mating amongst the previous clone series was carried out by NZ Forest Research Institute (NZFRI) staff in the late 1980s and the seedlings planted at three sites in 1993 as the 'Big Bang.' Only one trial at Tarawera forest survived the attack of Helicoverpa caterpillars. Selections were made there by the Radiata Pine Breeding Company (RPBC) (2003s)and OP seed and scion material were collected from each selection. The same procedure was followed in two other trials, the 1994/5-planted Wood Density and Dothistroma experiment ('2004' series), and in another OP trial from unselected parents ('2006' series). The new breeding programme is based on 360 forwards selections from these families, made on the basis of standardised trial assessments.

OP family tests of all 360 selections have been planted widely (11 trials in NZ and 7 trials in Australia). Selections were made at about 10 years and scions of all selections have all been grafted at the Purchase road (Amberley) archive.

The objectives were to rank all selections for BV for backwards selection for seed orchards. aControl cross seedlings will also be cloned for BP selection. Any development of a cloned BP has to be done from young CP seedlings, originating from crosses among the selections at the Purchase road archive.

CP families are being cloned to provide better estimates of breeding values (BVs) and to allow BP selection, with much higher heritabilities of clone means within families than within seedling families. Within-family selection on clone means will also not reduce genetic variation in the resulting BP as would selection among families. Since 2011, the RPBC has focused on selection traits $\mathrm{DBH}$, wood density and stiffness.

The RPBC is now cloning seedlings of crosses made in the Breeding Archive at Purchase road, Amberley. Cloning and later, within-family selection will allow selection of new BP trees without loss of genetic variation in the breeding programme which is now effectively closed. A combination of cloning and then within-family selection, will allow reliable forwards selection of BP clones as well as future orchard clones, and reduce time to deployment.

\section{Conclusion}

The factor that dominates this review is genetic variation. Without genetic diversity in the breeding population, there will be no gain from future generations of recurrent selection. Radiata pine has had the ability to propagate by rooted cuttings from the beginning of breeding, but the problem of maturation has prevented this propagation without ageing. The technology of 
propagation from young seedlings, essential for BP development, has now been mastered, as well as those of tissue culture and embryogenesis.

The other important element in this discussion is the need to select within cloned families for the future breeding population, losing no genetic variation in the process. There is also the capacity to select among and within cloned families to create the production population seed orchards.

Backwards selection to generate the breeding population was an important negative in this discussion, as it wastes genetic diversity, temporarily increases gain, but requires two cycles of breeding and testing, thus reducing gain per unit time.

Some researchers were concerned at the previous longterm reduction in genetic variability of the breeding population, while others were preoccupied by the immediate problems of getting gain and deploying the improved seed. There was a general ignoring of the problem in the NZ programme of loss of genetic variation in the breeding population. The excessive use of backwards selection, inevitably resulted in reduced genetic variation and lowered status number, which led to inbreeding in the BP.

An important contribution was made by those working on vegetative propagation of radiata who made a breakthrough in getting several cuttings from a young seedling to root and grow without serious 'c' effects, This made cloning of breeding populations feasible.

Shaw and Hood (1985) was the first paper to deal with cloning in a stochastic simulation. They pointed out what happens to genetic variance with among-families selection in advanced BPs versus maintaining genetic variation with clones in within-families selection. Perhaps the most influential paper was a stochastic simulation of gains in Norway spruce by Rosvall, Lindgren and Mullin (1998) who showed convincingly that solely within-family selection of clones over 10 generations gave continued gain and very little inbreeding, in spite of a big drop in status number. Additive genetic variance was little affected.

I was a strong convert to within-family selection with cloning, (Shelbourne et al. 1986) yet we didn't have an operational juvenile cutting multiplication method to create enough rooted cuttings per clone for a clonal test.

Deterministic simulation of gains from within-family selection in cloned and seedling OP, polycross and full-sib breeding populations also showed clearly how clones gave superior gains to seedlings up to and including $\mathrm{h}^{2} 0.5$.

The late Paul Jefferson in 2016, in an unfinished breeding management plan, really put cloning on the map. He planned the use of cloning in tree breeding of Pinus radiata. His objectives were the increase and acceleration of gain from forwards selection for seed orchards, and for within-family selection which would not reduce additive genetic variation in the BP. In a big industrial breeding programme, this application of cloning to within-family selection in the BP and realising gain in the seed orchards were both leading goals, and among the first in industrial breeding of pines.

\section{References}

Burdon RD, Shelbourne CJA (1971) Breeding populations for recurrent selection: conflicts and possible solutions. NZ J. For. Sci. 1(2):174-193.

Burdon RD, Shelbourne CJA (1974) The use of vegetative propagules for obtaining genetic information. NZ J. For. Sci. 4(2): 418-425.

Burdon RD, Shelbourne CJA, Wilcox MD (1977) Advanced selection strategies. Third World Consultation on Forest Tree Breeding, Canberra and Rotorua.

Burdon RD, Namkoong G (1983) Short note: Multiple populations and sublines. Silvae Genetica 32(5-6):221-222.

Burdon RD (1986) Clonal forestry and breeding strategies-a perspective. Proc. IUFRO Conf. Working Parties on Breeding Theory, Progeny Testing and Seed Orchards. pp. 645-659.Williamsburg, Virginia, p. 645.

Burdon RD (1989) Early selection in tree breeding: principles for applying index selection and inferring input parameters. Can. J. For. Res. 19: 499-504. https://doi.org/10.1139/x89-076

Burdon RD, Kumar S (2004) Forwards versus backwards selection: trade-offs between expected genetic gain and risk avoidance. NZ J. For. Sci. 34(1): 3-21.

Danell O (1993) Tree breeding strategy: are we too concerned conservationists but inefficient breeders? Proc. Nordic Group for Tree Breeding. Edinburgh, Scotland, 6-10 October 1993. Ed: Steve J. Lee.

Dungey HS, Brawner JT, Burger F, Henson M, Jefferson PA, Matheson AC (2009) A new breeding strategy for Pinus radiata in New Zealand and New South Wales. Silvae Genetica 58, 1-2. https://doi.org/10.1515/sg-2009-0004

Gea LD, Lindgren D, Shelbourne CJA, Mullin T (1997) Complementing inbreeding coefficient information with status number: implications for structuring breeding populations. NZJFS 27(3): 255-271.

Jayawickrama KJS, Carson MJ (2000) A breeding strategy for the New Zealand Radiata Pine Breeding Cooperative. Silvae Genetica 49, 2.

Jayawickrama KJS, Carson MJ (2000) A breeding strategy for the New ZealanRadiata Pine Breeding Cooperative. Silvae Genetica 49, 2.

Jefferson PA (2016) The breeding management plan. RPBC report Nov. 2016

Kumar S, (2006) Correlation between clonal means and seedling progeny means, and its implications for radiata pine breeding strategy. Canadian Journal of Forest Research 36:1968-1975. https://doi.org/10.1139/x06-082

Libby WJ (1964) Clonal selection and an alternative seed orchard scheme. Silvae Genetica 13:32-40.

Namkoong G, Snyder EB, Stonecypher RW (1966) Heritability and gain concepts for evaluating breeding systems such as seedling orchards. Silvae Genetica 15(3): 76-84.

Rosvall O, Lindgren D, Mullen TJ (1998) Sustainability, robustness and efficiency of a multi-generation breeding strategy based on within-family clonal selection. Silvae Genetica 47(5-6): 307- 321

Shaw DV, Hood JW (1985) Maximising gain per effort by using clonal replication in genetic tests. TAG 71:392-399. https://doi.org/10.1007/bf00251178

Shelbourne CJA (1969) Tree breeding methods. Technical Paper 55, Forest Research Institute, New Zealand Forest Service, Wellington.

Shelbourne CJA, Thulin IJ (1974) Early results from a clonal selection and testing programme with radiata pine. NZ J. For. Sci. 4 (2); 387-398.

Shelbourne CJA, Burdon RD, Carson SD, Firth A, Vincent TG (1986) Development plan for radiata pine breeding. Forest Research Institute, Rotorua, New Zealand.

Shelbourne CJA (1992) Genetic gains from different kinds of breeding population and seed or plant production population. South African Forestry Journal 160 March 1992. https://doi.org/10.1080/00382167.1992.9630411

Shelbourne CJA, Kumar S, Burdon RD, Gea LD, Dungey HS (2007) Deterministic simulation of gains for seedling and cloned main and elite breeding populations of Pinus radiata and implications for strategy. Silvae Genetica 56: 253-300. https://doi.org/10.1515/sg-2007-0037

Shelbourne CJA, Burdon RD, Dungey HS, Gea LD, Kumar S (2006) Revisiting the elite. RPBC/Ensis report Brain-storming workshop 28th February 2006. 